\title{
Le libre arbitre dans la pensée de R. Abraham bar Yehudah (élève de Hasdaï Crescas)
}

\author{
Shalom Sadik \\ The Van Leer Jerusalem Institute
}

El libre arbitrio en el pensamiento de R. Abraham bar Yehudá (discípulo de Ḥasday CRESCAS).- Análisis del pensamiento de R. Abraham bar Yehudá (Creta y Corona de Aragón, segunda mitad del siglo XIV) respecto del libre arbitrio. La primera parte del artículo muestra cómo al inicio de su libro (Eben Šetiyyá, 'Piedra fundamental') R. Abraham presenta una posición cercana a la opinión determinista de su maestro Hasday Crescas, poniendo de relieve que ya en el tercer cuarto del siglo XIV se sostenían ideas deterministas en la escuela de Crescas. La segunda parte del artículo muestra cómo en la continuación de su obra, R. Abraham presenta una opinión diferente, más cercana a la posición no-determinista común a la mayoría de los filósofos judíos medievales. El artículo concluye, explicando la contradicción del autor derivada de incluir en su obra dos opiniones diferentes tomadas de fuentes diversas, sin intentar elaborar un acuerdo entre ellas.

Palabras Clave: filosofía judía medieval; Ḥasday Crescas; libre arbitrio; determinismo; Abner de Burgos.

Free Will in the Thought of R. Abraham bar Yehuda (Student of Hasdai CresCAS). - The aim of this article is to analyze the opinion of R. Abraham bar Yehudah (Crete and the Crown of Aragon in the second half of the $14^{\text {th }}$ century) on the question of free will. The first part of the article describes how in the beginning of his book (Even Shetiyyah, 'Foundation Stone'), R. Abraham presents a position close to the deterministic thought of his well known teacher Hasdai Crescas, thus proving that in the third quarter of the $14^{\text {th }}$ century there already were deterministic views in the school of the later. The second part of the article demonstrates that in the continuation of this book R. Abraham presents a different opinion closer to the common non-deterministic position of the majority of Jewish medieval philosophers. The article concludes, explaining the contradiction, that in his book R. Abraham bar Yehuda brings in two different opinions from different sources without trying to build an accord between them.

KeYwords: Medieval Jewish Philosophy; Hasdai Crescas; Free Will; Determinism; Avner of Burgos.

\footnotetext{
*navitshalom@hotmail.com
} 
Rabbi Abraham bar Yehudah a vécu en Crête et en Espagne au cours de la deuxième moitié du $14^{\text {ème }}$ siècle. Sa pensée philosophique, exposée dans l'ouvrage Eben Shetiyyah ${ }^{1}$, n'a pratiquement pas fait l'objet d'étude systématique $^{2}$. Elle présente pourtant un double intérêt, tant pour la compréhension de la philosophie juive dans le monde méditerranéen au cours de la deuxième moitié du $14{ }^{\text {ème }}$ siècle, que pour la connaissance des courants de pensée dans le cercle des élèves de Hasdaï Crescas pendant cette même période ${ }^{3}$. Le but de cet article est d'exposer les opinions de R. Abraham sur l'un des principaux sujets où s'est révélée l'originalité de son maître Hasdaï, le libre arbitre.

\section{LA QUESTION DU LIBRE ARBITRE}

Hasdaï Crescas expose son opinion déterministe quant au libre arbitre humain dans la cinquième division de la deuxième partie de son livre, $\mathrm{Or}$ Adonai $^{4}$ :

\footnotetext{
${ }^{1}$ Sur le titre de ce livre voir E. LAwEe, «The Path of Felicity: Teachings and Tensions in Even Shetiyyah of Abraham ben Judah, Disciple of Hasdai Crescas», Mediaeval Studies 59 (1997), 183-223: note 1. Le livre a été publié dans S. RosenBerg, «Arba'a Turim of Rabbi Abraham bar Judah, Disciple of Don Hasdai Crescas» (héb.), Jerusalem Studies in Jewish Thought 3 (1983-1984), 525-621.

${ }^{2}$ Essentiellement l'étude de Lawee et l'édition de Rosenberg comprenant une courte introduction.

${ }^{3} \mathrm{R}$. Abraham a étudié à Barcelone lors de sont séjour en Catalogne vers 1375. À propos du rapport entre R. Abraham et le cercle de Hasdaï voir LAwEe, «The Path of Felicity», 211-221; RosenBERG, «Arba'a Turim», 527-528 et ses notes à cette édition. À propos de la relation entre la pensée de R. Abraham et celle de Hasdaï Crescas, ses deux chercheurs sont en désaccord: Rosenberg affirme que l'ouvre Eben Shetiyyah vient complètement de la pensée de Crescas dans les années 1370. Au contraire, Lawee pense que ce livre renferme les opinions propres de R. Abraham qui peuvent être différentes que celles de son maitre (nous reviendrons sur ce débat vers la fin de l'article). À propos du cercle des élèves de Hasdaï Crescas, voir N. OfIR, Rabbi Hasdai Crescas as a Philosophical Commentator on the Words of Chazal (héb.) (Ph.D. dissert., Jérusalem: The Hebrew University of Jerusalem, 1993), 321-349. Ce cercle comprenait des élèves et des collègues de Hasdaï Crescas comme Yosef Albo, Shem Tov Ibn Shaprout, Profayt Duran, Zerahyah ha-Levi (Ferrer Saladin), et Matityahu ha-Yitzhari, pour ne citer que les plus connues.

${ }^{4}$ Essentiellement Or Adonaï, éd. S. Fisher (Jérusalem, 1990), 205-225. Il existe une importante littérature moderne sur ce sujet, voir entre autres, A. RAvitZKY, «Crescas'
} 
ולזה הוא מבאר שאין בכל הטענות ההם מצד העיון ומצד התורה

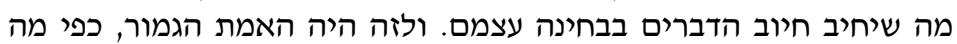

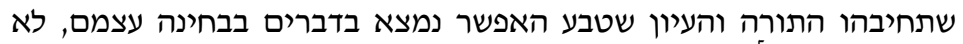

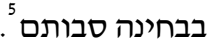

'Il est donc clair que rien dans ces arguments, qu'ils proviennent de la réflexion rationnelle ou de la Torah, ne prouve que les choses sont intrinsèquement nécessaires, mais uniquement qu'elles le sont par leurs causes' ${ }^{6}$.

Sa théorie se base sur la différence qu'établit l'auteur entre, d'une part, la liberté de l'homme par rapport à lui-même (מבחינת עצמו), et d'autre part, sa liberté en fonction des circonstances qui peuvent l'influencer (מבחינת סיבתו). Ces circonstances, intérieures et extérieures, obligeront l'homme à agir d'une manière définie. Selon Hasdaï Crescas, l'homme par lui-même, séparé des causes qui l'influencent est libre, mais les causes le poussent à utiliser sa liberté d'une manière déterminée. Cette opinion a, bien entendu, des répercussions sur de nombreux autres sujets. Par exemple, elle résout le problème du rapport entre la connaissance divine et le libre arbitre, car il n'y a pas de libre arbitre réel ${ }^{7}$. En ce qui concerne

Theory of Human Will: Development and Sources» (héb.), Tarbiz 51 (1982), 445-470; IDEM, Crescas' Sermon on the Passover and Studies in his Philosophy (héb.) (Jérusalem, 2005²), 34-68; J. Guttmann, Religion and Knowledge (héb.) (Jérusalem, 1979), 149168; S. Feldman, «Crescas’ Theological Determinism», Daat 9 (1982), 3-28; IDEM, «A Debate Concerning Determinism in Late Medieval Jewish Philosophy», Proceedings of the American Academy for Jewish Research 51 (1984), 15-54; Z. HARVEY, "On A. Ravitzky Crescas' Sermon on the Passover» (héb.), Tarbiz 58 (1989), 531-535; IDEM, «Comments on the Expression 'Feeling of Compulsion' in R. Hasdai Crescas» (héb.), Jerusalem Studies in Jewish Thought 4 (1985), 275-280; IDEM, R. Hasdai Crescas (héb.) (Jérusalem, 2010), 120-126; S. SADIK, Trinity and Determinism in the Thought of Abner of Burgos (héb., Ph.D. dissert., Ben Gurion University of the Negev, 2011), 282-286; IDEM, «Human Choice and Animal Will in Jewish Philosophy at the End of the Middle Ages» (héb.), Jewish Studies. An Internet Journal 9 (2010), 181-203: 199-202.

${ }^{5}$ Or Adonaï, éd. cit., 214.

${ }^{6}$ Traduction basée sur celle d'Eric SMILEvitch (trad.), Hasdaï Crescas, Lumière de l'Eternel (Paris-Strasbourg, 2010), 748.

${ }^{7}$ Sur la connaissance divine chez Hasdaï Crescas, voir L. STEIN, Die Willensfreiheit und ihr Verhältniss zur göttlichen Präscienz und Providenz bei den jüdischen Philosophen des Mittelalters (Berlin, 1882), 41-52; R. KrYGIER, À la limite de Dieu: L'énigme de l'omniscience divine et du libre arbitre humain dans la pensée juive (Paris, 1998), 179- 
la rétribution des bonnes et des mauvaises actions, celle-ci est définie comme la conséquence naturelle de l'acte.

R. Abraham décrit le processus psychologique de la décision humaine au début de son livre Eben Shetiyyah, lors de la comparaison entre la volonté divine d'une part et la volonté et le choix humains d'autre part:

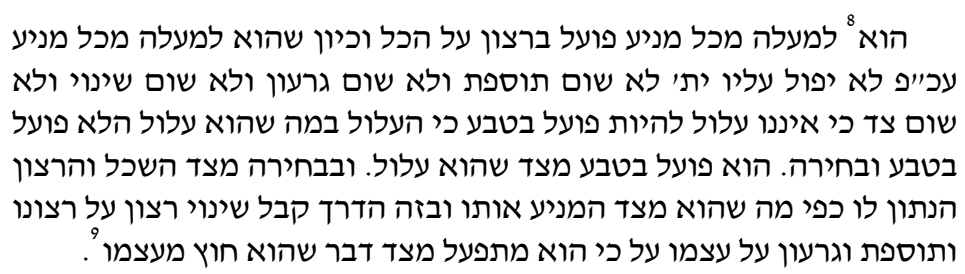

'Il [Dieu] est au-dessus de tous les instigateurs. Il agit volontairement sur tous, et étant donné qu'il est au-dessus de tous les instigateurs, de toute façon il ne Lui sera attribué [qu'Il soit béni] aucun rajout ou déficit, ni aucun changement, ni aucun parti pris car Il n'est pas un causé pour agir par nature. Car le causé, en tant que causé, agit selon la nature et le choix. Il agit selon la nature en tant qu'être causé, et selon le choix en tant qu'individu possédant un intellect et une volonté qui lui sont attribués comme tels par son investigateur. De cette manière, sa volonté est sujette au changement de volonté et lui-même [est sujet] au rajout et au déficit car il est mis en mouvement par un élément extérieur à lui-même'.

Dans ce passage, R. Abraham expose la différence qui existe entre les actes humains et les actions divines. Dieu est au-dessus de tous les instigateurs et sa volonté agit sur tout. La volonté divine n'est sujette à aucun rajout, ni à aucun manque ou changement. Dieu n'est pas causé

213; H. Kreisel, Maimonides' Political Thought: Studies in Ethics, Law, and the Human Ideal (New York, 1999), 435-439 et 452.

${ }^{8}$ L'auteur parle de Dieu. Ce passage compare la volonté divine à la volonté humaine pour expliquer la création du monde. Sur ce sujet voir Eben Shetiyyah, 536-539. Vers le début de ce passage, R. Abraham a déjà noté le fait que Dieu n'a pas de cause qui l'obligerait à agir ou l'empêcherait d'agir (מאין למעלה ממנו עלה שיכריחהו לעשות או שימנעהו) מלעשות).

${ }^{9}$ Eben Shetiyyah, 537-538. Dans la suite du texte R. Abraham décrit la volonté divine comme indépendante et n'agissant pas de manière naturelle (לעל בכלוונה וברצון בבחינת עצמו). (לא שהוא פועל בטבע). 
(עלול) et n'agit pas de manière naturelle. Pour comprendre la psychologie humaine, il est important de noter la relation qu'établit R. Abraham entre l'action de celui qui a une cause et l'action par nature et choix (בטבע (ובבחירה).

Celui qui est causé agit naturellement en tant que tel (מצד שהוא עלול). Il agit aussi selon son choix, car il possède un intellect et une volonté (מצד שד (השכל והרצון) causés eux même par leurs causes ${ }^{10}$. Ainsi, l'homme subit un changement et un ajout de volonté à sa volonté propre, contrairement à Dieu. L'homme agit par nature en tant que causé et par sa volonté qui est elle-même déterminée par une cause.

La volonté divine dépend entièrement de l'essence divine et n'a pas de cause extérieure. Les hommes, au contraire, agissent naturellement par choix et par volonté. La différence entre leurs actions naturelles et leurs actions par choix et volonté n'est qu'une différence de point de vue ${ }^{11}$. Les humains agissent naturellement car ils ont une cause et leurs actions ne sont qu'une conséquence des causes qui les précèdent. Mais par ailleurs, la même action peut être envisagée comme venant de leur volonté et de leur intellect car ils utilisent ces derniers lors du processus psychologique amenant aux choix.

Selon le fonctionnement de la psychologie humaine décrit dans ce passage, l'intellect et la volonté humaine ne sont pas les sources propres de l'action humaine mais les intermédiaires par lesquels les humains reçoivent et intègrent les causes, extérieures ou intérieures, avant leur choix. L'homme agit toujours naturellement, car il a toujours une cause. Lorsqu'il procède au choix, il se sert de son intellect et de sa volonté

${ }^{10} \mathrm{Il}$ est possible de comprendre les mots הנתון לו כפי מה שהוא מצד המניע אותו dans ce texte de deux manières différentes: 1) Ces mots parlent de la volonté qui est définie par la cause proche. 2) Ces mots parlent de l'intellect et de la volonté donnée par la nature (ou Dieu) à l'homme. Je pense que la première interprétation est plus probable car si le mot מתון parlerait de l'intellect et de la volonté, il devrait être au pluriel. De plus dans ce passage, le terme moteur מניע signifie la cause proche et pas Dieu comme cause générale du monde. De toute manière cette différence d'interprétation n'est pas cruciale pour notre sujet. En effet même selon la deuxième possibilité, la différence entre l'homme agissant par rapport à son choix et l'homme agissant comme ayant une cause reste une différence de point de vue uniquement. L'homme agit comme ayant une cause d'un certain point de vue et de part son choix d'un autre point de vue.

${ }^{11}$ Différence qualifiée par le terme hébreu מצד. 
pour analyser la situation dans laquelle il se trouve et décider. Mais sa décision n'en est pas moins naturelle car elle est également la conséquence de causes extérieures. R. Abraham ne mentionne jamais la liberté comme ayant son origine dans l'intellect, la volonté ou le choix humain. La différence entre la chute d'une pierre dans le vide et la décision d'un être humain réside dans la différence entre diverses lois naturelles, physiques ou psychologiques, mais les deux évènements sont naturels et ont une cause.

Un autre exemple de déterminisme psychologique se trouve dans la suite du premier chapitre d'Eben Shetiyyah, lors d'une comparaison entre la volonté divine et la volonté humaine:

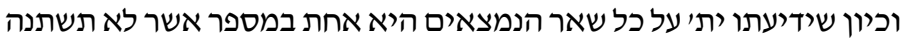

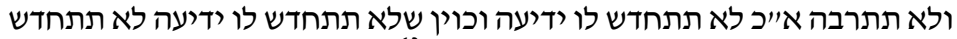

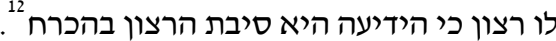

'Et comme Sa connaissance, qu'Il soit béni, de tout ce qui existe est une et unique ${ }^{13}$, sans nouvelle acquisition, ni ne change ni ne se multiplie, il n'aura donc pas de nouvelle connaissance et comme il n'aura pas de nouvelle connaissance, Sa volonté restera la même car la connaissance est la cause obligatoire de la volonté'.

Dans ce passage, R. Abraham note que la connaissance de Dieu ${ }^{14}$ est immuable car elle englobe dès le départ tout ce qui existe. Dans l'une de ses phrases, l'auteur décrit la relation entre la connaissance et la volonté, clés de la psychologie humaine. La connaissance est la cause menant nécessairement à la volonté. Le rapport entre la cause psychologique, ici la connaissance, et la volonté humaine est un rapport obligatoire. Selon R. Abraham, le changement de connaissance n'explique pas seulement le changement de volonté, il le détermine ${ }^{15}$. C'est la raison pour laquelle R. Abraham peut

${ }^{12}$ Eben Shetiyyah, 545-546. Dans la suite du texte R. Abraham mentionne un exemple et se sert du terme 'obligé' (חייב) pour définir la relation entre la nouvelle connaissance et la volonte qui provient de cette nouvelle connaissance.

${ }^{13}$ Littéralement 'est une de par le nombre'.

${ }^{14}$ Dans la deuxième partie de l'article, nous analyserons en détail l'opinion de notre auteur sur la connaissance divine.

${ }^{15}$ A propos des différents modes de causalité dans la pensée d'Aristote voir R. SorABJI, 
démontrer que Dieu n'a pas de volonté en se basant sur le fait que sa connaissance est statique. Dans une situation donnée, la volonté de l'homme et ses actions sont complètement déterminées par les causes extérieures.

Selon les deux textes vus plus haut, R. Abraham adopte donc une position proche de celle de son maitre Hasdaï Crescas ${ }^{16}$. Chez ces deux philosophes, le processus psychologique humain est défini par les capacités de l'âme humaine, volonté et intellect. Ces capacités fonctionnent de manière tout à fait naturelle et déterminée. Mais dans Eben Shetiyyah, se trouvent des passages dans lesquelles la liberté humaine est envisagée différemment.

Dans la première partie d'Eben Shetiyyah ${ }^{17}$, R. Abraham analyse différentes questions ayant trait à la connaissance divine. Celle-ci peut appréhender plusieurs domaines sans être divisée et connaître le futur sans changer le cours des évènements ${ }^{18}$. R. Abraham se tourne vers le problème de la relation entre la connaissance divine et la liberté humaine:

$$
\begin{aligned}
& \text { ואמנם התרת הספק השני והוא שאנו צריכין לדעת ידיעתו ית' בנבראים }
\end{aligned}
$$

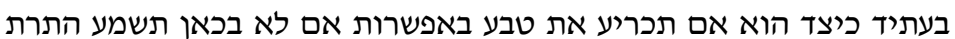

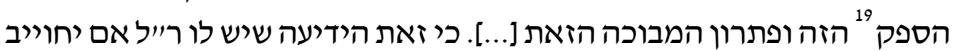

Necessity, Cause, and Blame - Perspectives on Aristotle's Theory (New York, 1980).

${ }^{16}$ En cela, R. Abraham est différent de la plupart des autres élèves de Hasdaï Crescas, qui n'ont pas adopté la position de leur maitre sur ce sujet. À propos de l'opinion de Yosef Albo sur la psychologie humaine voir S. SADIK, «Freedom of Choice in the Thought of R. Yosef Albo» (héb.), Jewish Studies. An Internet Journal 11 (2012), 139-151. Sur l'opinion de Matityahu ha-Yitzhari, voir A. Ravitzky, «The Paradoxical Concept of Free Will in Matityahu ha-Yitzhari» (héb.), in From Rome to Jerusalem (Jérusalem, 1998), 239-256.

${ }^{17}$ Eben Shetiyyah, 539-555 (sur ce sujet voir aussi p. 566). Dans ce passage R. Abraham adopte une terminologie proche de celle d'Abraham Ibn Ezra. À propos de l'opinion de ce philosophe sur la connaissance divine, voir Y. CoHEN, The Philosophical Thought of Rabbi Abraham Ibn Ezra (Ramle, 1996), 242-268.

${ }^{18}$ R. Abraham adopte sur ce sujet la position d'Averroès. Plusieurs penseurs juifs médiévaux avaient déjà adopté cette opinion, par exemple R. Moïse de Narbonne. À propos de son opinion sur ce sujet voir M. Hayoun, La théologie et la philosophie de Moïse de Narbonne (Tübingen, 1989), 110-123. Et les sources citées dans SADIK, «Human Choice and Animal Will», 197 (note 60).

${ }^{19}$ Dans le texte publié il est marqué הפסק par erreur. 
'Quant à la solution du deuxième doute, nous devons savoir si Sa connaissance [la connaissance par Dieu] qu'Il soit béni, de ce qui va se passer dans le futur des créatures, annulera la nature de la possibilité de changement ou non? Ici tu écouteras la solution de ce doute et le dénouement de cette incertitude [...]. Car cette connaissance qu'Il a, j'entends par là si les choses doivent obligatoirement suivre Sa connaissance des créatures, qu'Il soit béni, malgré cela [cette connaissance] n'annulera pas la possibilité de changement. Car Dieu, qu'Il soit béni, n'est ni la cause ni la raison de l'accident et de la possibilité. Car cette chose [la possibilité et l'accident] est attribuée à la nature en tant que nature, ce qui signifie qu'il [celui qui a deux possibilités] est prêt à recevoir une option possible et peut lui-même annuler [l'autre] option possible selon son bon vouloir. Et Dieu, qu'Il soit béni, n'annulera pas une des options possibles, sauf en cas de récompense ou de punition'.

Dans ce passage, l'auteur pose la question classique de la relation entre la connaissance divine et la liberté humaine. Dans l'optique de Hasdaï Crescas, le déterminisme psychologique élimine la question d'emblée. En effet, si l'homme est obligé, de par ses causes, à utiliser sa volonté d'une manière connue d'avance, il est naturel que Dieu connaisse l'avenir car la possibilité réelle est inexistante. Selon cette optique, Dieu a tout assigné par la création des lois déterminant la volonté humaine dans chaque situation pratique. Malgré les points communs entre la psychologie de Hasdaï Crescas et celle de R. Abraham, ce dernier se distingue de son maître sur le sujet de la connaissance divine.

Selon R. Abraham, la connaissance divine du futur existe mais n'a pas d'influence sur le déroulement des actions dans le monde réel. Il retourne ainsi à la position la plus courante dans la pensée juive médiévale ${ }^{22}$

20 אמר probablement par erreur dans l'édition de Shalom Rosenberg.

${ }^{21}$ Eben Shetiyyah, 548.

${ }^{22}$ Cette optique était d'abord celle de Saadya Gaon (voir Krygier, À la limite de Dieu, 41-45); puis celle d'un nombre important de sages dont Bahya Ibn Paquda (voir 
en affirmant qu'il y a une différence entre le déroulement des actions dans le monde, provoquées par leurs différentes causes, et la connaissance de Dieu qui est indépendante de ces causes. La connaissance de Dieu n'impose donc pas la possibilité de l'une des deux alternatives (חא תכריע (האפשרות), car elle n'est pas la cause qui détermine laquelle des options peuvent théoriquement avoir lieu et se réaliser. Cette cause est la cause naturelle, qui décide par elle-même (בעצמו), selon sa volonté, quelle sera la situation choisie. Cela est vrai sauf dans le cas où Dieu empêchera le déroulement naturel du processus pour punir ou récompenser ${ }^{23}$.

Selon R. Abraham, la liberté humaine est réelle, l'homme est la cause qui, grâce à sa volonté, décide comment une situation donnée évoluera. Cette liberté est maintenue, sauf en cas d'une intervention divine. Dans la suite de ce texte, $\mathrm{R}$. Abraham établit clairement que la rétribution des actions de l'homme par Dieu et les commandements divins seraient vidés de leur sens sans liberté humain réelle:

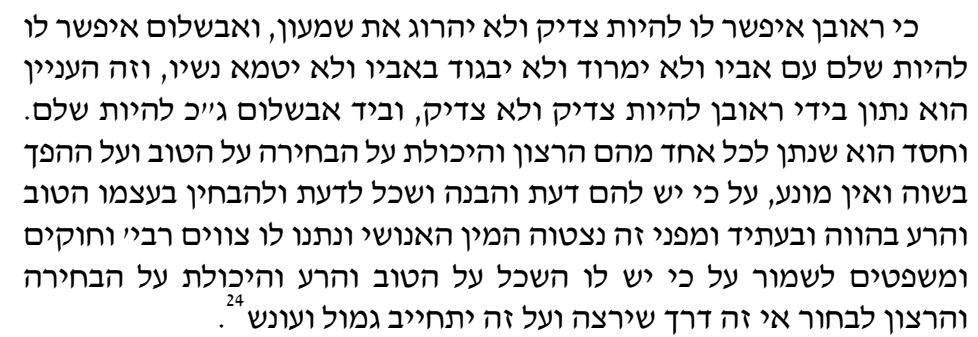

Krygier, À la limite de Dieu, 49-50); L. KNOLLER, Das Problem der Willensfreiheit in der älteren jüdischen Religionphilosophie (Breslau, 1882), 29-35; ALtmann, «Free Will and Predestination in Saadia, Bahya, and Maimonides», 44-47; Maïmonide, Guide des Egares III, 19-23. Sur l'optique de Maïmonide voir S. SADIK, «Maimonides Mechanism of Choice» (héb.), AJS Review 38 (2014), א- , et le résumé des différentes interprétations offertes dans la recherche moderne.

${ }^{23}$ Dans la suite du texte (Eben Shetiyyah, 548-555) R. Abraham explique que la rétribution des actions humaines ne peut exister dans un monde déterminé par Dieu et que l'homme a une capacité (יכולת) réelle de décider de ses actes. La possibilité qu'à Dieu d'annuler la liberté humaine est aussi mentionnée par Maimonide dans le Traité des Huit Chapitres.

${ }^{24}$ Eben Shetiyyah, 551-552. À propos de la liberté humaine réelle, voir aussi pp. 554555. Il est intéressant de noter que dans ce texte, R. Abraham interprète les dires de R. Aquiba (הכל צפוי והרשות נתונה, Abot III, 15) comme exprimant un paradoxe: Dieu sait tout mais sa connaissance n'oblige en aucune manière les bonnes ou les mauvaises actions des 
'Car Ruben avait la possibilité d'être un juste et ne pas tuer Siméon, et Absalon avait la possibilité d'être en bon terme avec son père, de ne pas se révolter, de ne pas le trahir et de ne pas souiller ses femmes. Et il est dans le pouvoir de Ruben d'être juste ou injuste, et dans le pouvoir d'Absalon d'être en bon terme avec son père. Et ceci vient de la bonté [de Dieu] qui a donné à chacun d'entre eux la volonté et la capacité de choisir le bien et le contraire, de manière égale, sans empêchement. Donc ils ont la raison, la compréhension et l'intellect pour raisonner et distinguer par eux même le bien du mal dans le présent et le futur. C'est pour cela que le genre humain a reçu de multiples commandements, lois et ordonnances auxquels obéir. Car il à l'intellect [capable de distinguer] le bien du mal, la capacité de choisir et la volonté de décider quel chemin prendre. Et de ceci découlent nécessairement la récompense et la punition'.

Dans ce passage, R. Abraham dit que Dieu a donné la possibilité à chacun d'être juste ou pécheur. Cette possibilité vient de la bonté divine qui a octroyé à chacun, de manière équivalente, la volonté et la capacité du choix, bon ou mauvais. Dans la suite du texte, R. Abraham explique que les hommes ont la connaissance, la compréhension (דעה והבנה) et l'intellect qui leur permet de différencier le bien du mal. La capacité des hommes à choisir entre le bien et le mal, à analyser diverses possibilités grâce à leur intellect sont les raisons pour lesquelles Dieu a donné à l'homme des commandements et le récompense ou le punit selon ses actes.

Dans ces deux derniers passages, R. Abraham définit expressément la volonté et le choix de l'homme comme libres, et développe une théorie psychologique permettant cette liberté. Selon lui, la liberté humaine dépend des facultés intellectuelles qui permettent de différencier le bien du mal ainsi que de la volonté, qui a la capacité de faire des choix.

hommes. Cette interprétation est tout à fait contraire à celle de Hasdaï Crescas (Or Adonaï II, 5, 3, pp. 216-217) qui interprète cette mishnah comme montrant que tout ce qui se passe dans le monde est organisé (מסודר) par Dieu, tandis que l'homme dispose de sa liberté par rapport a lui-même (בבחינת עסמר) Dans la suite de son explication, Hasdaï Crescas interprète la fin de la mishnah comme exprimant son opinion concernant la rémunération des actions humaines. La rémunération est la conséquence de l'acte commis par l'homme. Cette opinion contredit celle de R. Abraham selon laquelle les commandements n'auraient aucun sens sans l'existence du libre arbitre. 
Ces deux derniers textes contredisent donc les deux premiers qui ont été étudiés plus haut. Dans les deux premiers textes, R. Abraham décrit une psychologie humaine déterminée par ses causes et qui se sert de son choix d'une manière bien définie. Au contraire, dans les deux derniers textes, l'auteur expose un processus de choix au cours duquel l'homme possède un intellect lui permettant de différencier le bien du mal et une volonté libre de trancher d'un côté ou de l'autre. Comment expliquer ces points de vue contradictoires exprimés par R. Abraham?

Il existe deux explications vraisemblables. Premièrement, il est possible d'interpréter les écrits traitant de la liberté réelle à la lumière des premiers textes définissant la position déterministe. R. Abraham a mentionné deux points de vue différents. En premier lieu, l'homme est envisagé par rapport à sa cause et agit de manière naturelle et déterminée. En deuxième lieu, il est examiné par rapport au choix humain et à son intellect qui sont libres, bien que déterminés par leur cause. Il serait donc possible d'interpréter les textes parlant de liberté humaine comme se concentrant essentiellement sur le point de vue interne du choix humain tout en ne contredisant pas le second point de vue $\mathrm{e}^{25}$, dans lequel l'homme resterait déterminé.

Cette interprétation, qui a le mérite de respecter la cohérence de la pensée de l'auteur, ne peut, à mon avis, être acceptée. Les deux derniers textes cités décrivent la psychologie humaine comme complètement libre ${ }^{26}$. Il est donc totalement impossible de les interpréter selon les textes déterministes. En effet, si la volonté humaine n'est pas complètement libre comment serait-il possible de dire que la récompense et la punition divines proviennent de la liberté humaine. Hasdaï Crescas a donné une toute autre interprétation de la récompense divine. Selon lui la récompense ou la punition sont une conséquence nécessaire de l'action et pas une rétribution ${ }^{27}$.

${ }^{25}$ Cette interprétation rendrait l'opinion de R. Abraham presque identique à celle de son maître.

${ }^{26}$ Logiquement la deuxième possibilité devrait être l'interprétation des premiers textes décrivant une psychologie déterministe à la lueur des seconds. Mais la description de l'homme comme cause et comme agissant par choix interne uniquement selon un point de vue particulier me semble empêcher une telle interprétation.

${ }^{27}$ Or Adonaï, 213. Hasdaï Crescas compare la personne punit pour avoir enfreint les commandements divines a un homme se faisant bruler par le feu après y avoir mis la main involontairement. On ne peut parler d'injustice dans les deux cas car il s'agit de la conséquence naturelle de l'action. 
Ensuite, il est envisageable que les deux opinions exposées dans le texte d'Eben Shetiyyah proviennent de deux sources différente, assemblées par R. Abraham et insuffisamment modifiées pour exposer une théorie psychologique unique et uniforme. Selon cette interprétation, les premiers textes montrent l'influence de Hasdaï Crescas sur R. Abraham. Voulant exposer la différence entre la volonté humaine et la volonté divine, ce dernier se serait servi de la théorie de Crescas décrivant une divinité agissant selon sa nature propre contrairement aux hommes, déterminés par des causes extérieures. Concernant la volonté divine, R. Abraham aurait adopté la théorie la plus courante dans le judaïsme médiéval ${ }^{28}$, théorie prônant le libre arbitre de l'homme ainsi que la connaissance divine. Il n'aurait pas jugé nécessaire de modifier ses différentes sources pour présenter une vision unique de la psychologie humaine.

\section{CONCLUSION}

Rabbi Abraham a certes été influencé par l'opinion déterministe de Hasdaï Crescas sur la question du libre arbitre ${ }^{29}$. Mais cette influence n'a pas été la seule. Il s'est inspiré de l'opinion de son maître pour, par exemple, accentuer la différence entre la dépendance humaine vis-à-vis des causes et l'indépendance divine. Mais concernant d'autres sujets tels que la relation entre la volonté humaine et la connaissance divine, il a préfèré suivre des voies plus traditionnelles ${ }^{30}$.

${ }^{28} \mathrm{R}$. Abraham n'a pas non plus adopté la position de son maître sur la question des attributs divins et de la connaissance divine (Eben Shetiyyah, 532, 541-557, 566 et 617). Etant donné que la question de la connaissance divine est en relation avec celle des attributs divins, la connaissance étant elle-même un attribut divin, il est logique que notre auteur ait tiré ses opinions sur ce sujet d'autres sources que de l'opinion originale de Hasdaï Crescas. Sur l'opinion de Crescas à propos de cette question voir H. A. WolfsON, «Crescas on the Problem of Divine Attributes», Jewish Quarterly Review [NS] 7 (1916), 1-44 et 175-121; I. EPSTEIN. «Das Problem des göttlichen Willens in der Schöpfung nach Maimonides, Gersonides und Crescas», Monatsschrift für Geschichte und Wissenschaft des Judenthums 75 (1931), 335-347.

${ }^{29}$ Son maitre étant lui-même sous l'influence de l'apostat Abner de Burgos. Pour un resumé des diverses opinions dans la recherche moderne concernant la relation entre Hasdaï Crescas et Abner sur ce sujet voir SADIK «Human Choice and Animal Will», 200 (note 74).

${ }^{30}$ Son opinion sur les attributs divins, proche de celle de Maimonide (sur ce sujet voir Eben Shetiyyah, 555-558, ainsi que Z. HARVEY, «Bewilderments in the Theory of 
La dynamique interne du cercle des élèves de Hasdaï Crescas est éclairée d'une lumière nouvelle. Tout d'abord, il est important de noter que R. Abraham est l'un des seuls élèves de Crescas à avoir adopté, du moins en partie, la théorie déterminisme de son maître. Le fait que R. Abraham ait tempéré la théorie déterministe de Hasdaï Crescas par l'utilisation d'autres sources confirme ce que l'on sait déjà du cercle de Crescas. Ses membres se servaient d'un grand nombre de sources et la plupart d'entre eux avaient des opinions plus traditionnelles ${ }^{31}$ que Hasdaï Crescas ${ }^{32}$.

Ensuite, il est intéressant de noter que déjà à l'époque où R. Abraham étudiait dans le cercle de Crescas, il existait un courant déterminisme. Il est donc très probable que l'influence de l'opinion déterministe d'Abner sur Hasdaï Crescas ait été antérieure à ce que pensent certains chercheurs modernes $^{33}$. Car notre étude de la pensée de R. Abraham montre que déjà dans les années 1370 des idées déterministes étaient présentes dans ce cercle.

L'utilisation par R. Abraham de théories non déterministes n'apprend rien sur l'opinion de Hasdaï Crescas à l'époque où R. Abraham fut son élève. Tous les élèves de Crescas dont la pensée a été l'objet d'une analy-

Attributes of Hasdai Crescas» [héb.], Proceedings of The Israel Academy of Sciences and Humanities 8 [1997], 133-144: 143) lui permettait de soutenir l'existence d'un paradoxe entre la connaissance divine et la connaissance humaine. Selon l'opinion de Hasdaï Crescas, un tel paradoxe ne peut exister.

${ }^{31}$ Tant traditionnel du point de vue religieux, comme pour la question du libre arbitre, que conservateur du point de vue philosophique, comme pour leur adoption de la physique d'Aristote. Il faut noter par exemple que la définition de l'infini selon R. Abraham (Eben Shetiyyah, 533-534 et 543-544) est essentiellement aristotélicienne et n'adopte pas la nouvelle définition de Hasdaï Crescas. À propos de l'opinion de Crescas sur ce sujet voir T. LEVY, «L'infini selon R. Hasdaï Crescas (1340-1412)», in Inquisition et pérennité, éd. D. Banon (Paris, 1992), 161-166; N. L. RABINOvitch, «Rabbi Hasdai Crescas (1340-1410) on numerical infinities», Isis 61 (1970), 224-230.

${ }^{32}$ Par example, à propos de la definition du lieu chez Hasdaï Crescas et Yosef Albo, voir SADIK, «Freedom of Choice in the Thought of R. Yosef Albo» (héb.), 139-151.

${ }^{33}$ Le débat moderne sur ce sujet est essentiellement entre Zeev Harvey et Aviezer Ravitzky. Selon ce dernier les deux dernier chapitres (chapitres V-VI) s'occupant du libre arbitre son parallèle au sermon de Hasdaï Crescas sur Pessah. Il conclue que ses deux chapitres, influencés par Duns Scot, on été écrits avant les quatre autre plus déterministes et influencé par Abner de Burgos. Notre analyse de la pensé de R. Abraham renforce la critique de Harvey (établissant qu'il n'est pas logique que Hasdaï Crescas ait connue Abner après Duns Scot) sur l'opinion de Ravitzky. 
se moderne, ont refusé de suivre l'opinion déterministe de leur maitre. Il est donc probable que R. Abraham, lui aussi, ait voulu retourner à des opinions plus traditionnalistes sur ce sujet. Il faut donc voir R. Abraham comme un penseur éclectique plutôt qu'un fidèle interprète de la pensée de Hasdaï Crescas à l'époque où il fut son élève $\mathrm{e}^{34}$.

Recibido: 21/12/2012

Aceptado: 23/04/2014

${ }^{34}$ Nos conclusions supportent donc l'opinion de Lawee qui vois le livre Eben Shetiyyah comme dévoilant la pensé propre de R. Abraham. Contrairement à l'opinion avancée par Rosenberg, selon laquelle cette œuvre dévoile uniquement les opinions de Hasdaï Crescas dans la fin des années 1370. 